\title{
Experimental Study of Photonuclear Reactions of Light Nuclei with NewSUBARU $\gamma$-ray Beam
}

\section{Tatsushi Shima*}

Research Center for Nuclear Physics, Osaka University, 10-1 Mihogaoka, Ibaraki, Osaka 567-0047, Japan

E-mail: shima@rcnp.osaka-u.ac.jp

\section{Yasuki Nagai}

Nuclear Science and Engineering Directorate, Japan Atomic Energy Agency, 2-4 Shirakata-Shirane, Tokai-mura, Naka-gun, Ibaraki 319-1195, Japan

\section{Hiroaki Utsunomiya, Hidetoshi Akimune}

Department of Physics, Konan University,

8-9-1 Okamoto, Higashinada, Kobe, Hyogo 658-8501, Japan

\section{Shuji Miyamoto, Ken Horikawa, Takayasu Mochizuki}

Laboratory of Advanced Science and Technology for Industry, University of Hyogo,

3-1-2 Kouto, Kamigori, Ako-gun, Hyogo 678-1205, Japan

\begin{abstract}
A new laser Compton-backscattered $\gamma$-ray source at the NewSUBARU synchrotron radiation facility was applied to the experimental studies of the photonuclear reactions relevant to astrophysical nucleosynthesis. The performance of the NewSUBARU $\gamma$-ray source and the current status of the experiment on the photodisintegration of ${ }^{4} \mathrm{He}$ are presented.
\end{abstract}

10th Symposium on Nuclei in the Cosmos

July 27 - August 12008

Mackinac Island, Michigan, USA

\footnotetext{
*Speaker.
} 


\section{Introduction}

Photonuclear reactions on light nuclei at low energies play essential roles in various processes of the nucleosynthesis, and therefore precise nuclear data of those reactions are indispensable to study the origin of elements and the evolutions of stars and the universe. The photonuclear reactions also provide important information about the analogous neutrino-induced nuclear reactions by the weak neutral current [1], which are supposed to play critical roles in the dynamics of corecollapse supernovae $[2,3,4]$ and nucleosynthesis occurring in the neutrino-driven wind $[5,6,7]$.

New-generation $\gamma$-ray sources based on the laser Compton-backscattering (LCS) method are expected to be powerful tools for high-precision experiments of the photonuclear reactions in the energy region relevant for astrophysical nucleosynthesis because of their good performances of the monochromatic and variable energies, large poralizations of nearly $100 \%$, small angular dispersions, and little background $\gamma$-rays. Recently a new LCS $\gamma$-ray source was developed at the NewSUBARU synchrotron radiation facility of the Laboratory of Advanced Science and Technology for Industry at the University of Hyogo, Japan [8], and was employed for experimental studies of photonuclear reactions on light nuclei relevant to astrophysical nucleosynthesis. In what follows the outline of the NewSUBARU LCS $\gamma$-ray source and the ongoing experiment on the photonuclear reactions on ${ }^{4} \mathrm{He}$ are presented.

\section{NewSUBARU LCS $\gamma$-ray source}

The NewSUBARU is a race-track shaped electron storage ring with the maximum electron energy of $1.5 \mathrm{GeV}$. The LCS $\gamma$-rays are generated via the head-on collision of an electron beam and a laser light, and the $180^{\circ}$ backscattered $\gamma$-rays are extracted with a collimator made of a $10 \mathrm{~cm}$ thick lead brick. A nice feature of the NewSUBARU LCS $\gamma$-ray source is the very small background due to the bremsstrahlung, because the laser light and the electron beam collide in a long straight section of the storage ring. Fig. 1 shows the foreground and the background $\gamma$-ray pulse height spectra measured with a large volume $(76 \mathrm{~mm} \times 76 \mathrm{~mm} \times 180 \mathrm{~mm})$ GSO scintillation counter with and without injection of the laser light, respectively.

Since the NewSUBARU can provide $\gamma$-rays in the energy range from a few $\mathrm{MeV}$ up to 40 $\mathrm{MeV}$ by changing the electron beam energy and the laser wavelength, it is useful to measure the excitation functions of the photonuclear reactions in the energy regions from the particle thresholds up to beyond the nuclear giant resonances. The total intensity of $2 \times 10^{5}$ photons/MeV/s and the energy spread of $8.7 \%$ in FWHM were achieved for the maximum $\gamma$-ray energy of $E_{\gamma}=17 \mathrm{MeV}$ using a $3 \mathrm{~W} \mathrm{Nd}: \mathrm{YVO}_{4}$ laser and a $1 \mathrm{GeV}$ electron beam with a storage current of $200 \mathrm{~mA}$ [9].

\section{Photodisintegration of ${ }^{4} \mathrm{He}$}

The nuclear response of ${ }^{4} \mathrm{He}$ to the spin dipole excitations is of special importance concerning the effect of the $v$ - $\alpha$ inelastic scattering to the dynamics and the nucleosynthesis in neutrino-driven wind [5]. So far, the dipole excitation of ${ }^{4} \mathrm{He}$ has been investigated by measuring the cross sections of the photodisintegration of ${ }^{4} \mathrm{He}$ as well as their inverse radiative capture reactions of ${ }^{3} \mathrm{H}(\mathrm{p}, \gamma){ }^{4} \mathrm{He}$ 


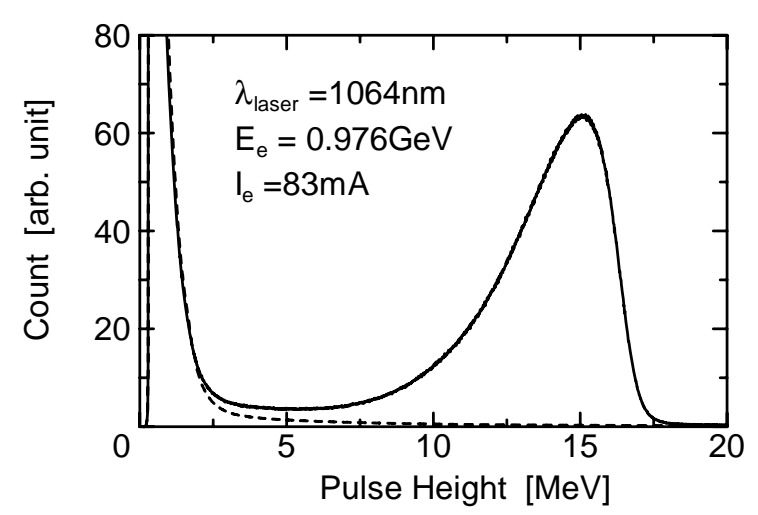

Figure 1: $\gamma$-ray pulse height spectra measured with a GSO scintillation counter. The solid curve and the dashed curve indicates the spectra with laser power of $3.53 \mathrm{~W}$ and $0 \mathrm{~W}$, respectively.

and ${ }^{3} \mathrm{He}(\mathrm{n}, \gamma){ }^{4} \mathrm{He}$ (see references in [10]). In the energy region above $E_{\gamma} \sim 40 \mathrm{MeV}$, most of the existing data are in agreement with each other. On the other hand, the data show discrepancies as large as $50 \sim 100 \%$ below $E_{\gamma} \sim 40 \mathrm{MeV}$, which may be caused by the influence of low-energy background $\gamma$-rays and/or uncertainties in the determination of the experimental parameters like the geometrical acceptance of a detector, the effective thickness of the target, and the absolute intensity of the incident beams. With the above situation in mind, we performed a measurement using new techniques of a quasi-monochromatic LCS $\gamma$-ray beams and a time projection chamber (TPC) [11], which could detect the charged particles from the photonuclear reactions with an efficiency of nearly $100 \%$ and a solid angle of $4 \pi$, because it contained helium gas as an active target. In our former experiment using the LCS $\gamma$-ray source at the National Institute of Advanced Industrial Science and Technology (AIST) in Tsukuba, Japan, the cross sections of both the $(\gamma, \mathrm{p})$ and $(\gamma, n)$ reactions of ${ }^{4} \mathrm{He}$ were found to increase in the measured $\gamma$-ray energy region up to $30 \mathrm{MeV}$, being quite different from many of the previous experimental data as well as the latest theoretical calculation [10]. In order to investigate the feature of the excitation functions of the photonuclear reactions on ${ }^{4} \mathrm{He}$, we made a new experiment in the extended energy range up to $37.2 \mathrm{MeV}$ at NewSUBARU. Fig. 2 shows a schematic view of the experimental setup. The LCS $\gamma$-rays with the maximum energies ranging from $29.5 \mathrm{MeV}$ to $37.2 \mathrm{MeV}$ were generated using a $1064 \mathrm{~nm}$ laser light and electron beams with the energy of 1.3-1.46 GeV. As demonstrated by Fig. 3, the particle tracks of the photonuclear reactions on ${ }^{4} \mathrm{He}$ were clearly observed. A preliminary result for the ${ }^{4} \mathrm{He}(\gamma, \mathrm{n})^{3} \mathrm{H}$ reaction cross section is shown by Fig. 4. The present result for the energy region below $E_{\gamma}=31 \mathrm{MeV}$ is found to be in good agreement with our previous one at $E_{\gamma}=30 \mathrm{MeV}$. Above $E_{\gamma}=31 \mathrm{MeV}$, the present result suggests the peak of the excitation function at around $E_{\gamma}=$ $33 \mathrm{MeV}$, which is quite different from the results from many of the other experiments including the latest $(\gamma, n)$ measurement using the tagged photon beam at the MAX-lab in Lund University [12]. To solve this problem, further experiments are in progress both in the MAX-lab and in the NewSUBARU. It is also important to compare with the calculations based on recent theories of the few-nucleon systems in order to elucidate the mechanism of the photodisintegration of ${ }^{4} \mathrm{He}$. 


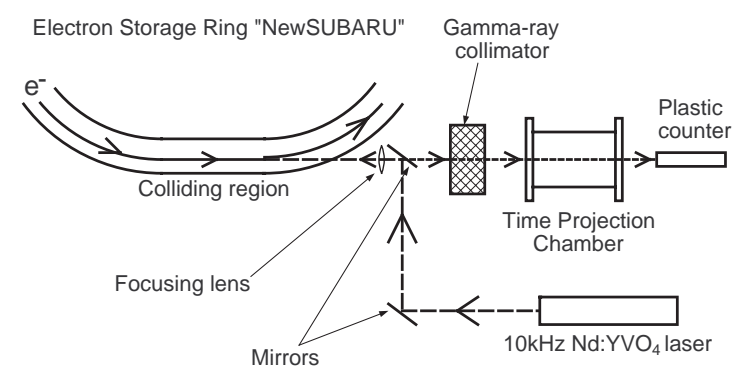

Figure 2: Schematic view of the experimental setup at the NewSUBARU LCS facility.
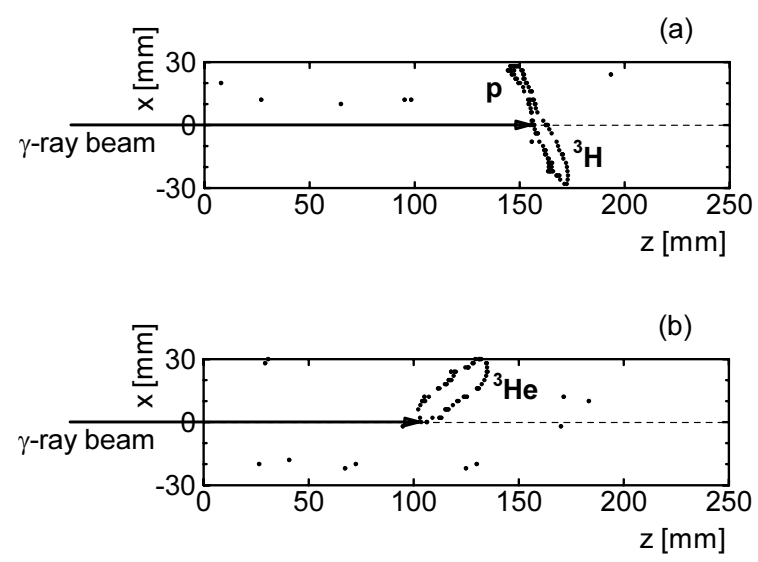

Figure 3: Examples of the observed tracks of the charged particles emitted by the photodisintegration of ${ }^{4} \mathrm{He}$. (a): ${ }^{4} \mathrm{He}(\gamma, \mathrm{p})^{3} \mathrm{H}$ and (b): ${ }^{4} \mathrm{He}(\gamma, \mathrm{n})^{3} \mathrm{He}$. The boxes show the side view of the effective volume of the TPC. The tracks are indicated by the dots corresponding to the drift times of the leading and trailing edges of the signals measured by the cathode wires of the TPC.

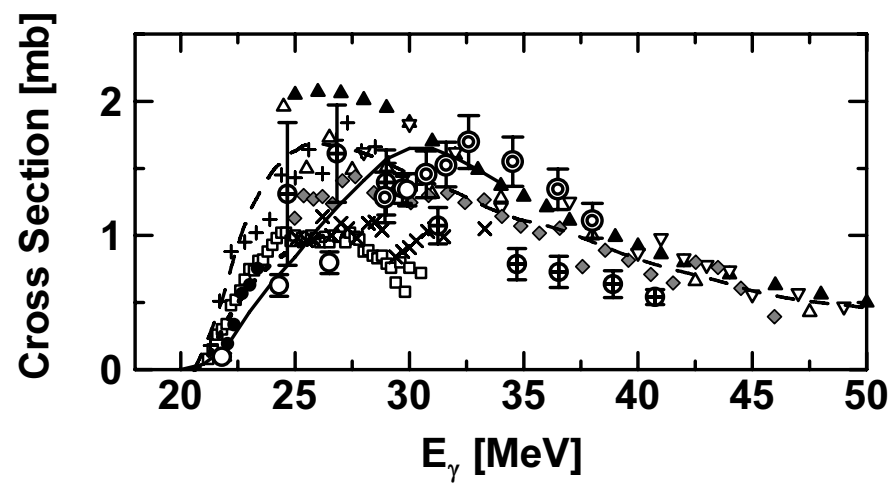

Figure 4: Summary of the ${ }^{4} \mathrm{He}(\gamma, \mathrm{n})^{3} \mathrm{H}$ cross section data. The double circles indicate the preliminary results from the present work. The open circles denote our previous data measured at AIST [10]. The crossed circles are the data measured with a tagged photon beam [12]. The other symbols indicate the previous data according to the definition used in Fig. 1 in Ref. [10]. The solid, dashed, and dotted curves denote the theoretical calculations taken from Refs. [13], [14], [15], respectively. 


\section{Summary}

The laser Compton backscattering method provides high-quality quasi-monochromatic $\gamma$-ray beams which are suitable for high-precision experiments on low-energy photonuclear reactions relevant to astrophysical nucleosynthesis. The NewSUBARU LCS facility was developed recently, and was shown to have a nice feature of little background $\gamma$-rays. The photonuclear reactions on ${ }^{4} \mathrm{He}$ were successfully measured with use of the NewSUBARU LCS $\gamma$-ray beam in order to explore the nature of the dipole excitations of ${ }^{4} \mathrm{He}$ which is relevant to the neutrino-induced nucleosynthesis in core-collapse supernovae. The following experimental studies with the NewSUBARU LCS $\gamma$-ray source are planned ;

- photonuclear reactions on light nuclei $\left({ }^{3} \mathrm{He},{ }^{12} \mathrm{C},{ }^{16} \mathrm{O}\right.$, etc.) relevant to the stellar evolutions and r-process nucleosynthesis,

$\cdot(\gamma, \mathrm{n})$ reactions of long-lived radioactive nuclei, and

$\cdot(\gamma, p)$ and $(\gamma, \alpha)$ reactions of p-nuclei with the photo-activation method.

\section{Acknowledgments}

The authors would like to thank Profs. T. Kajino, K. Sumiyoshi, and Dr. T. Yoshida for fruitful discussions on the effect of the $v$ - $\alpha$ interactions in the nucleosynthesis in supernovae. This work was supported in part by Grant-in-Aid for Specially Promoted Research of the Japan Ministry of Education, Science, Sports and Culture and in part by Grant-in-Aid for Scientific Research of the Japan.

\section{References}

[1] S.E. Woosley, D.H. Hartmann, R.D. Hoffman, and W.C. Haxton, Astrophys. J. 356 (1990) 272.

[2] W.C. Haxton, Phys. Rev. Lett. 60 (1988) 1999.

[3] S.E. Woosley et al., Astrophys. J. 433 (1994) 229.

[4] H.-T. Janka and E. Muller, Astron. Astrophys. 306 (1996) 167.

[5] B.S. Meyer, Astrophys. J. 449 (1995) L55.

[6] T. Yoshida, M. Terasawa, T. Kajino, and K. Sumiyoshi, Astrophys. J. 600 (2004) 204.

[7] A. Heger et al., Phys. Lett. B606 (2005) 258.

[8] K. Aoki et al., Nucl. Instr. Meth. in Phys. Res. A516 (2004) 228.

[9] S. Miyamoto et al., Radiation Meas. 41 (2006) S179-S185.

[10] T. Shima et al., Phys. Rev. C72 (2005) 044004.

[11] T. Kii, T. Shima, T. Baba, and Y. Nagai, Nucl. Instr. Meth. in Phys. Res. $\mathbf{A 5 5 2}$ (2005) 329.

[12] B. Nilsson et al., Phys. Lett. B626 (2005) 65; B. Nilsson et al., Phys. Rev. C75 (2007) 014007.

[13] J.T. Londergan and C.M. Shakin, Phys. Rev. Lett. 28 (1972) 1729.

[14] W. Sandhas et al., Nucl. Phys. A631 (1998) 210c.

[15] S. Quaglioni et al., Phys. Rev. C69 (2004) 044002. 